\title{
Detecção de Falhas e Fraturas em Reservatórios de Baixa Permeabilidade Utilizando Atributos Geométricos
}

\author{
José Eduardo M. Lira (Petrobras), Rogério de A. Santos (Petrobras), Frank C. Bulhões (Petrobras), Gleidson D. Ferreira
} (Petrobras), Arnaldo Tanaka (Petrobras), Julio G. Carvalho (Petrobras), Adriano César Barreto (Petrobras)

\section{Copyright 2018, SBGf - Sociedade Brasileira de Geofísica}

Este texto foi preparado para a apresentação no VIII Simpósio Brasileiro de Geofísica, Salinópolis, 18 a 20 de setembro de 2018. Seu conteúdo foi revisado pelo Comitê Técnico do VIII SimBGf, mas não necessariamente representa a opinião da SBGf ou de seus associados. E proibida a reprodução total ou parcial deste material para propósitos comerciais sem prévia autorização da SBGf.

\section{Resumo}

Um dos principais desafios exploratórios na análise de reservatórios de gás, que possuam baixa permeabilidade é a detecção e distribuição espacial de elementos estruturais como falhas e fraturas. Estas estruturas podem fornecer informações das principais rotas de migração, indicando regiões com variações acentuadas na permeabilidade dos sistemas rochosos envolvidos. A utilização de atributos geométricos é bastante útil na detecção sísmica destes elementos estruturais. Apresenta-se, neste trabalho, uma estratégia de processamento interpretativo para detecção de falhas e fraturas utilizando um fluxo de pré-condicionamento do dado sísmico para a melhor performance combinada de dois atributos de coerência: MSA (Minimal Similarity Accumulation) e Curvatura. O fluxo corresponde a análises interpretativas dos resultados de uma sequência de filtros e inversões com o objetivo de atenuar ruídos e melhorar a resolução dos dados sísmicos para a posterior aplicação do atributo MSA em conjunto com a curvatura máxima. Tal procedimento é aqui descrito ilustrativamente em dados sísmicos terrestres no campo de Marizeiro, bacia Potiguar (Brasil) e da Bacia de Neuquén (Argentina). Os resultados mostram melhorias na resolução do dado, na detecção e visualização de descontinuidades geométricas, auxiliando a interpretação sísmica estrutural.

\section{Introdução}

Um dado sísmico pode ser compreendido como uma representação de um modelo de sub-superfície terrestre, através de diversas sequências de interfaces, contínuas e descontínuas, muitas das quais associadas a estruturas geológicas, como falhas e fraturas. A qualidade do imageamento sísmico de tais estruturas é inversamente proporcional ao nível de ruído dos dados. Um dos objetivos do processamento sísmico é produzir imagens que honrem a geologia de sub-superfície e que possam ser interpretadas pelos exploracionistas. A otimização do imageamento e posicionamento de falhas e fraturas possui um papel fundamental na exploração de campos gás, cujos reservatórios possuem baixa permeabilidade. Tal otimização tem repercussões econômicas diretas em projetos exploratórios, no posicionamento e projeto de poços, no desenvolvimento de reservatórios, e planejamento de injeção e recuperação.
A coerência sísmica é uma medida de similaridade matemática que pode ser aplicada traço a traço no dado sísmico e serve como indicativo do grau de continuidade de eventos. Torna-se assim um poderoso atributo para separar áreas de descontinuidades, que estão em geral associadas a zonas mais fraturadas ou mais permeáveis.

Atributos de coerência vêm sendo utilizados há longo tempo para detecção e mapeamento de falhas e fraturas, Gersztenkorn et al (1999), citam que os primeiros trabalhos utilizando medidas de coerência sísmica datam, da década de 60, citando Taner et al (1969) que utilizaram a coerência para calcular os espectros de velocidade. O trabalho de Bahorich et al.(1995) é uma importante referência ao apresentar uma metodologia que adota uma combinação entre correlações cruzadas traço a traço (Inline e crossline), gerando o chamado cubo de coerência. Marfurt et. al. (1998) estenderam tal trabalho para um cenário multitraço, visando reduzir a influência do ruído nos resultados. Posteriormente, Gersztenkorn e Marfurt (1999) apresentaram um algoritmo de auto-estrutura da matriz de covariância, incorporando vários filtros e esquemas de interpolação para tratar ruídos e problemas de alias.

Neste trabalho, para definição otimizada de descontinuidades estruturais sísmicas, apresenta-se um fluxo para atenuação de ruídos seguido pela aplicação de um atributo de coerência, denominado MSA (Figueiredo et al,2014 \& Figueiredo et al.2016). Como précondicionamento dos dados, sugere-se a aplicação de uma sequência de filtros, Curvelet (Neelamani et al. 2008) e um filtro de suavização orientado por estrutura (FSOE) (Machado et al., 2015). Os resultados de aplicação de tal fluxo são ilustrados em dois volumes sísmicos empilhados, de Marizeiro e de Neuquén, ambos contendo gás em reservatórios de baixa permeabilidade.

\section{Campo de Marizeiro - Bacia Potiguar - Brasil}

A bacia Potiguar tem seu desenvolvimento iniciado no início do Cretáceo, resultando da abertura do Atlântico Sul (Françolin e Szatmari, 1987). A Bacia Potiguar ocupa uma área de aproximadamente $48.000 \mathrm{Km}^{2}$, onde 21.500 $\mathrm{km}^{2}$ em terra e $26.500 \mathrm{~km}^{2}$ em mar. Ela faz parte de um sistema de rift intracontinental que abriu de Sul para Norte no sul do Atlântico Sul e de Oeste para Este no ramo do Oceano Equatorial (Matos, 1992). O Campo de Marizeiro é um reservatório de baixa permeabilidade composto por arenitos e conglomerados com baixa permo-porosidade e pode ser definido como um reservatório do tipo tight gas sands. Devido a tais características, o mapeamento das zonas com maior 
densidade de fraturas é fundamental para embasar a locação de poços exploratórios e de desenvolvimento.

\section{Bloco Rio Neuquén - Bacia de Neuquén - Argentina}

O bloco Rio Neuquén está localizado a aproximadamente $30 \mathrm{~km}$ do NNE da cidade de Neuquén, bacia de Nequén, Argentinas, possuindo uma área de $397,59 \mathrm{~km}^{2}$. Reservatórios jurássicos de gás, com baixa peremabilidade, são encontrados na Formação Lajas (areias amalgamadas intercaladas por pelitos) e Punta Rosada (areias e argilas fluviais, deltaicas e estuarinas) que se configuram em plays de gás de centro de bacia. Para a produção destes reservatórios são fundamentais a compreensão da distribuição espacial e densidade das fraturas naturais existentes.

\section{O Pré-condicionamento de dados sísmicos}

A multiplicidade de um levantamento sísmico é um dos principais fatores que controlam a qualidade de dados sísmicos terrestres. Por multiplicidade, ou cobertura, entenda-se o número de vezes que determinado ponto em sub-superfície é amostrado em um levantamento sísmico. Quanto maior esse número, melhor será a razão sinal-ruído que está diretamente ligada à qualidade do dado. Algoritmos desenhados para detecção sísmica de fraturas são muito sensíveis à presença de ruídos. Como na sua maioria, tais algoritmos estão, basicamente, correlacionando, ou comparando, determinado traço com os seus vizinhos (Bahorich et al., 1995), a atenuação de ruídos é crítica para a sua performance.

Para atenuar a ruídos coerentes, no presente trabalho foi aplicado filtro do tipo Curvelet (Neelamani et al. 2008, op cit.) seguido por um filtro de suavização orientado por estrutura (FSOE) (Machado et al. 2015, op cit.), que busca melhorar a continuidade de refletores. Para destacar planos de falhas e possíveis fraturas, foi aplicada um processo de deconvolução iterativa - Iterdec (Rosa 2010), visando aumentar a resolução. Após o dado passar pelo fluxo da Figura 1, rodamos os algoritmos de MSA e Curvatura.

Para analisarmos os resultados do fluxo de précondicionamento, apresentamos na Figura 2, seções sísmicas do dado original, painel superior e do dado após a filtragem, painel inferior. É possível perceber a redução de ruídos e a melhoria na visualização dos eventos. Após os filtros, aplicamos a deconvolução iterativa (Rosa,2010) e ilustramos o resultado na Figura 3, onde apresentamos no painel superior o dado após os filtros de Curvelet e FSOE e no painel inferior, a mesma seção após o Iterdec. Nos círculos amarelos, pode-se observar o ganho de qualidade e de resolução dado, originário do ganho no conteúdo de frequências devido à deconvolução. É importante destacar que os exemplos mostrados nas figuras 2 e 3 , referem-se ao dado do campo de Marizeiro. Devido à alta qualidade do dado de Rio Neuquén, não foi necessária a aplicação do fluxo de pré-condicionamento antes do MSA.
Algoritmos de Curvatura são bastante utilizados para determinação de áreas fraturadas em exploração de reservatórios do tipo tight-gas de baixa permeabilidade.

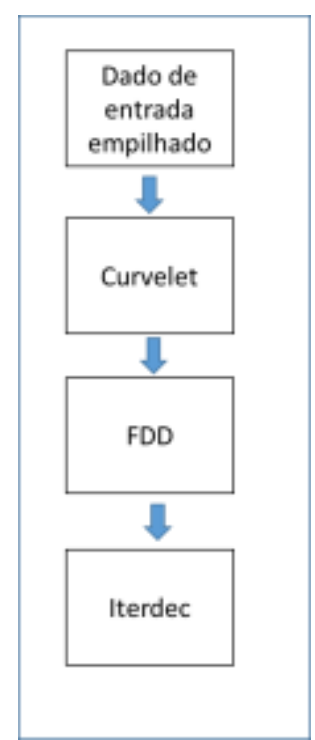

Figura 1. Esquema do fluxo de processamento utilizado para preparar 0 dado para a posterior aplicação do atributo para identificação de falhas e fraturas (MSA). O filtro Curvelet na atenuação de ruídos, o FDD na melhoria da continuidade dos refletores e a deconvolução iterativa (Iterdec) para o aumento da resolução.

\section{$O$ atributo MSA}

Em atividades sísmicas de E\&P, o mapeamento de falhas e fraturas é fundamental, principalmente para reservatórios de gás. Além da importância de definição de rotas de migração, as acumulações ocorrem em regiões vizinhas onde a densidade de fraturas é maior. Horizontes homogêneos e contínuos são representados no dado sísmico como eventos com um maior grau de coerência entre amplitudes de traços próximos. Visualmente, estes eventos em áreas mais homogêneas mostram uma variação menor na amplitude de traços contíguos, quando comparados com eventos em áreas mais fraturadas. Por outro lado, a resposta sísmica em áreas fraturadas apresenta, via de regra, menor coerência matemática com relação à resposta de regiões menos fraturadas. É baseado nessas premissas genéricas que os algoritmos de identificação de falhas e fraturas buscam caracterizar descontinuidades estruturais. 

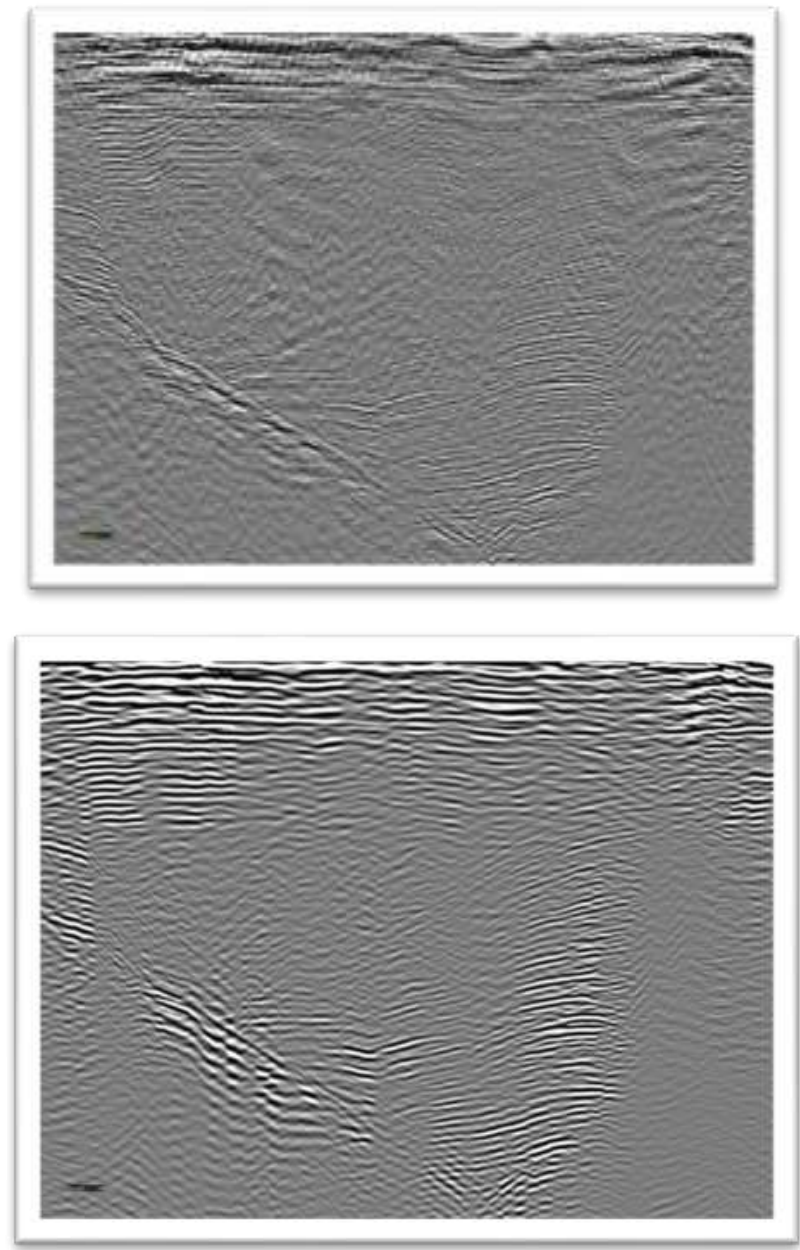

Figura 2. Seção do dado original (painel superior) e a mesma seção (painel inferior) após 0 précondicionamento com filtragem Curvelet e FDD no Campo de Marizeiro. Nota-se a melhora na qualidade da seção e visualização dos eventos, após a aplicação dos filtros para atenuação dos ruídos.

O atributo MSA (Minimal Similarity Accummulation) (Figueiredo et al. 2014) aplicado a dados 3D pode ser descrito em três etapas: i) Agrupamento de voxels baseado em janelas verticais, visando principalmente a atenuação de ruídos no dado de entrada; ii) Cada voxel passa a representar um agrupamento correspondente a uma função que é então definida para medir a similaridade no espaço dos agrupamentos e iii) $\mathrm{O}$ atributo MSA é então calculado pela soma da similaridade mínima entre os voxels vizinhos. Neste trabalho, foi utilizada a versão otimizada do MSA proposta por Figueiredo et al (2016).
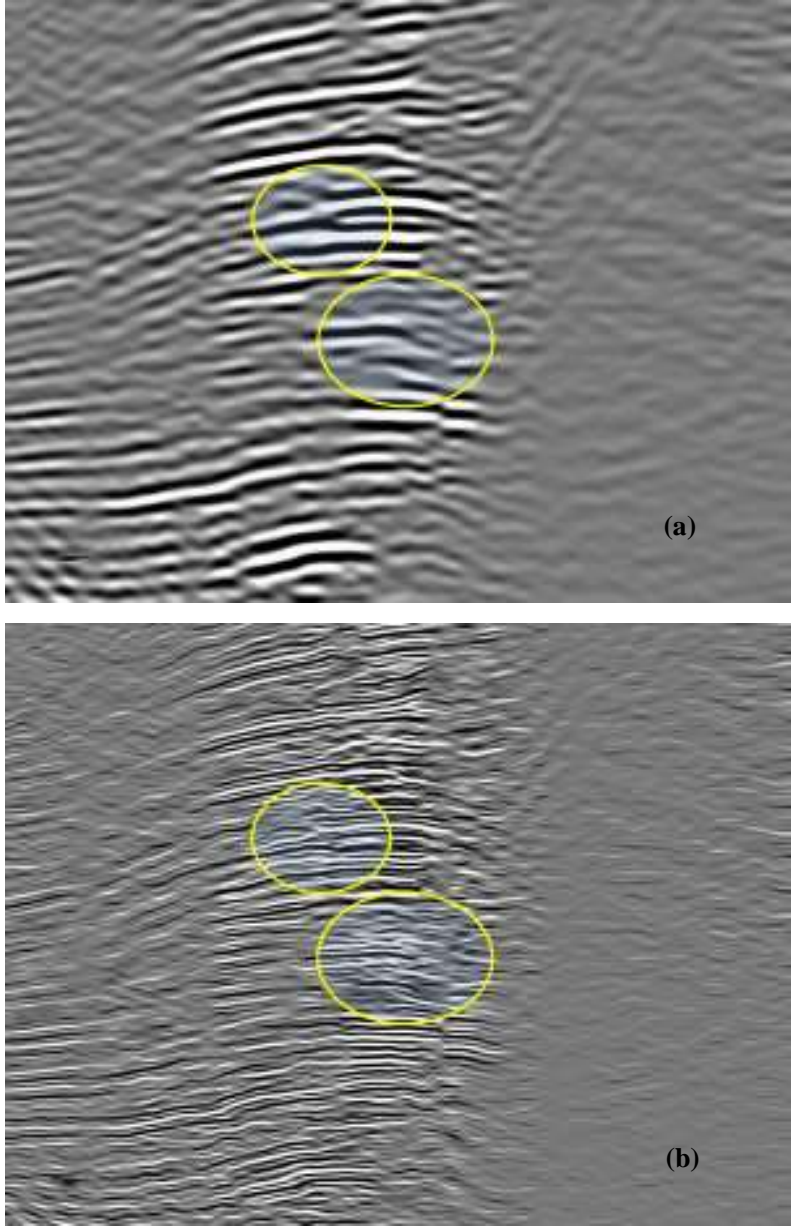

Figura 3. (a) Dado com filtragem Curvelet e FDD. (b) Aplicação de filtros e deconvolução iterativa no Campo de Marizeiro. Nota-se o aumento da resolução e a melhor definição dos planos de falhas em destaque nos círculos amarelos.

\section{Resultados}

O procedimento descrito anteriormente, foi aplicado no volume empilhado de rio Neuquén. Para ilustrar, na Figura 4 (a) destaca-se um trecho de uma linha do dado original de entrada e em 4 (b), o dado após a aplicação do atributo MSA. Observa-se uma melhor visualização das estruturas de pequenas falhas, em destaque na elipse amarela. Estas estruturas são quase imperceptíveis no dado original podendo ser confundidas com ruído. A visualização de uma região mais falhada também no destaque na elipse em vermelho. 


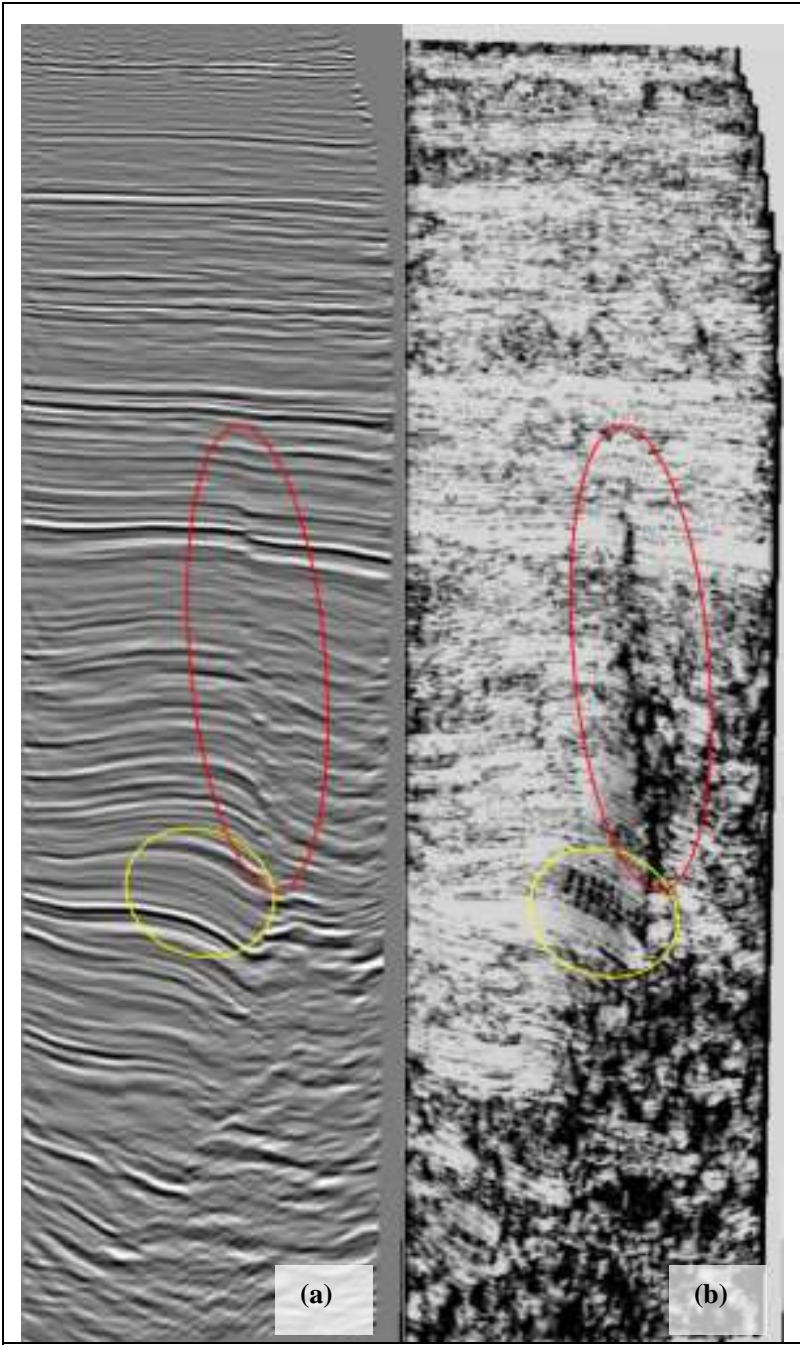

Figura 4. (a) Seção sísmica original do dado de Rio Neuquén, (b) mesma seção com aplicação do MSA. Observa-se uma melhor visualização de pequenas falhas, destaque no contorno em amarelo, no dado com MSA. Uma zona de falhas mais extensa, contorno em vermelho, pode ser melhor identificada no dado com MSA.

Devido à qualidade do dado de rio Neuquén, não foi necessário a aplicação do pré-condicionamento. No caso do volume do campo de Marizeiro, aplicamos o fluxo de filtros. As figuras 2 e 3 mostram o ganho em termos de qualidade do dado, atenuação de ruídos, e a melhora na resolução.

Na figura 6, apresenta-se uma fatia de tempo do volume sísmico de rio Neuquén, com a curvatura máxima sobre o MAS. Observa-se uma boa concordância entre ambos os atributos. A Curvatura máxima em vermelho pode indicar zonas mais curvadas e/ou fraturadas.

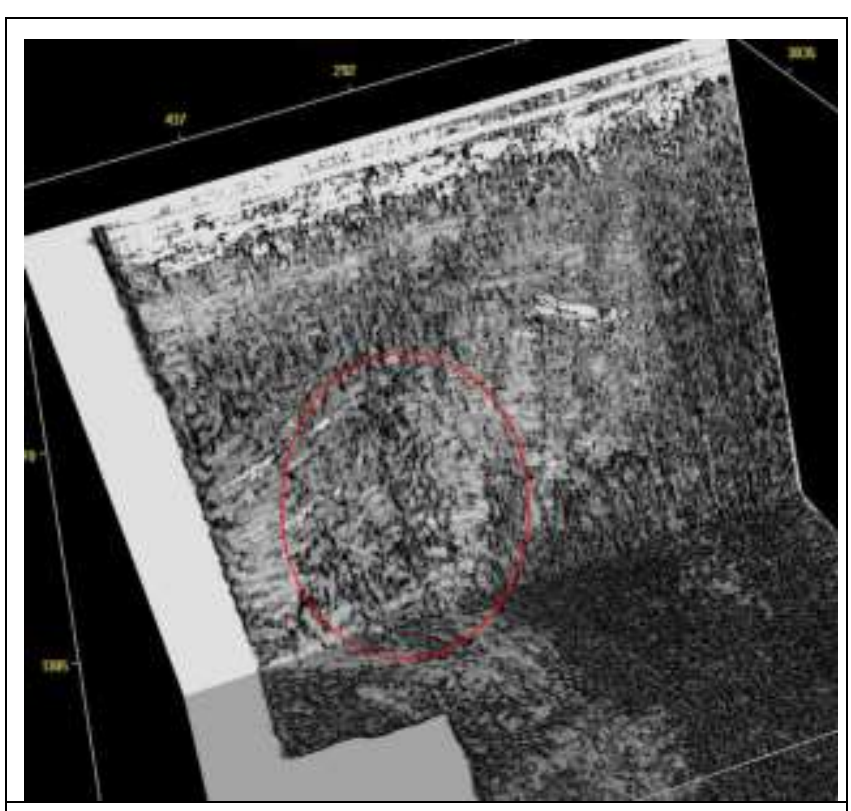

Figura 5. Volume de MSA do dado de Marizeiro após o pré-condicionamento. Falhas/fraturas no embasamento são melhor identificadas com a aplicação do MSA (ver detalhe no contorno em vermelho).

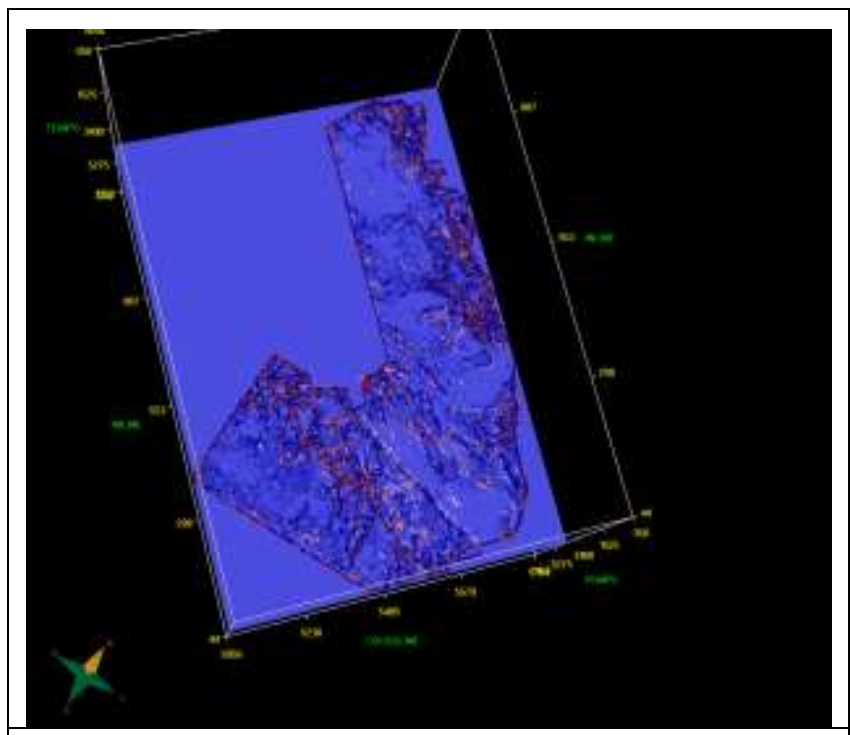

Figura 6. Fatia de tempo do dado de rio Neuquén mostrando os atributos Curvatura máxima (vermelho) e MSA. Observa-se uma concordância entre ambos, no mapeamento de lineamentos. 


\section{Comentários Finais}

Apresentamos um fluxo de pré-processamento para aplicação de um atributo, MSA, para identificação de falhas e fraturas. $\mathrm{O}$ atributo mostrou-se bastante efetivo melhorando a visualização de falhas como mostrado na figura 4, Bacia Neuquén, e na figura 5, Campo de Marizeiro, bacia Potiguar. $O$ dado de rio Neuquén, que possui uma qualidade superior ao dado de Marizeiro, mostrou o potencial do atributo. Os resultados em Marizeiro, após a aplicação do pré-codicionamento, foram bastante positivos, indicando regiões com maior densidade de fraturas. A melhor identificação de falhas permite a construção de um modelo estrutural mais realista, contribuindo para uma a redução do risco exploratório em bacias terrestres. O Iterdec (Rosa, 2010) propiciou uma melhoria significativa na resolução e se mostrou ferramenta importante para definição de planos de falhas e fraturas.

A aplicação de um fluxo de pré-condicionamento no dado sísmico é fundamental para se obter o máximo de atributos de coerência.

\section{Bibliografia}

Bahorich, M. e Farmer, S. (1995) " 3-D seismic discontinuity for faults and stratigraphic features: The coherence cube." The Leading Edge, 14(10), pp: 10531058.

Figueiredo, A.M., Faustino, G.M., Peçanha,J.P., Silva, P.M. e Gattass, M. (2014) "Minimal Similarity Accumulation Attribute for Fault Enhancement", $84^{\text {th }}$ SEG Annual Meeting - Denver, EUA.

Figueiredo, A.M., Peçanha,J.P., Faustino, G.M., Silva, P.M. e Gattass, M. (2016) " High Quality Horizon MappingUsing Clustering Algorithms" - 78th EAGE Conference and Exhibition, Viena, Austria

Françolin, J.B.L., Szatmari, P. (1987) Mecanismo de rifteamento da porção oriental da margem norte brasileira. Revista Brasileira de Geociências, v. 17, p.196207.

Gersztenkorn, A. e Marfurt, K.J. (1999). "Eigenstructurebased coherence computations as an aid to 3-D structural and stratigraphic mapping." Geophysics, Vol. 64, № 5 , pp:1468-1479.

MATOS, R.M.D. (1992) Deep seismic profiling, basin geometry and tectonic evolution of intracontinental rift basins in Brazil. U.S.A.: Cornel University (Tese de Doutorado).

Neelamani,R. , Baumstein, A.I., Gillard,D.G. Hadidi,M. T. e Soroka, W.L. (2008). "Coherent and random noise attenuation using the curvelet transform." The Leading Edge,27(2),240-248.

Rosa, A.L.R., (2010) "Análise do Sinal Sísmico" Sociedade Brasileira de Geofísica, 668 pp.
Taner, M.T. e Koehler, F.., (1969) Velocity spectra- Digital computer derivation and application of velocity functions, Geophysics, 34, 859-881 